\title{
Letter
}

\section{Namibia and Botswana's zebra migration: still the longest, but for how long? A reply to Schapira et al.}

We welcome Schapira et al.'s (2016) news of another longdistance migration in Africa, of white-eared kob Kobus kob leucotis in Ethiopia and South Sudan. As we (Naidoo et al., 2016) and they note, little documentation on this migration exists; when our paper was published in 2014 the only peer- approximately $20-30 \mathrm{~km}$, although this metric clearly underestimates the kob migration's overall extent.

We expect that Schapira et al.'s continued research on the kob migration will uncover additional insights; our own zebra research has documented longer and more spatiallyvariable movements since 2014 (Fig. 1). We hope further wildlife migrations are discovered, including those that eventually take the mantle of Africa's longest.

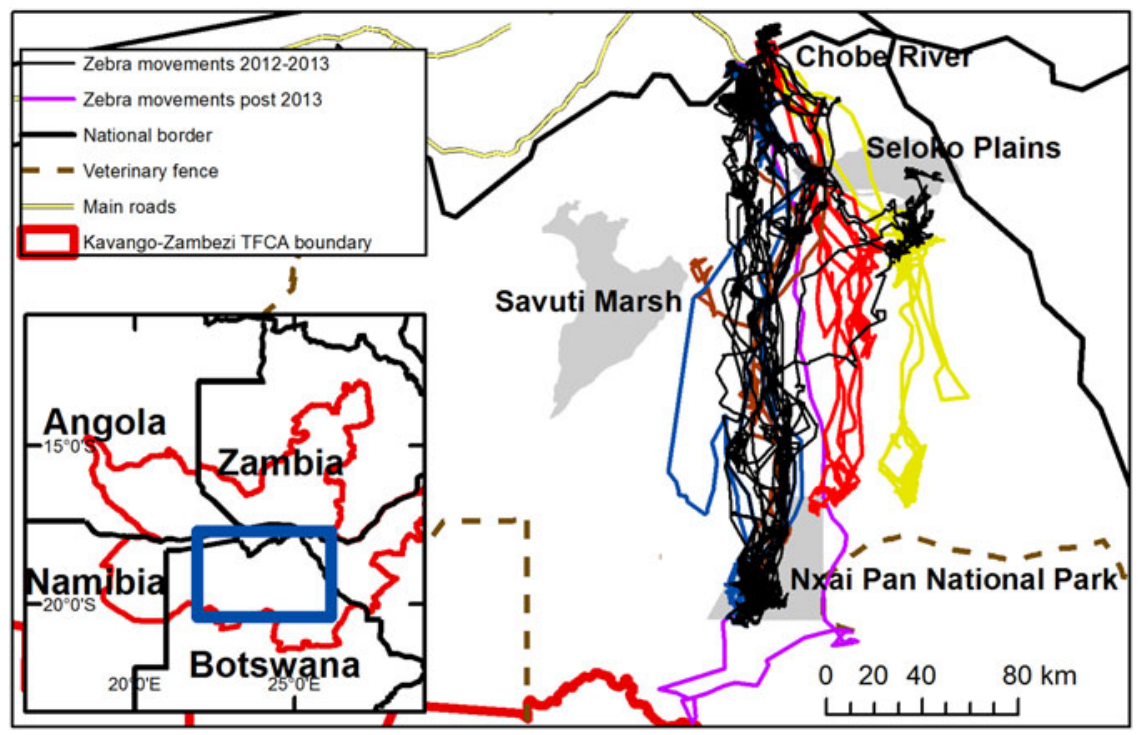

FIG. 1 The migration of Burchell's zebra Equus quagga in Namibia and Botswana, with data from global positioning system (GPS) collars used in our original 2014 publication represented by black lines, subsequent GPS data in other shades (different shades indicate different individuals), and with the Chobe River and Nxai Pan National Park endpoints indicated.

reviewed reference was Harris et al. (2009), describing a round-trip distance for kob of $300-400 \mathrm{~km}$.

Schapira et al. suggest that we incorrectly presented the Burchell's zebra Equus quagga migration in Namibia and Botswana as Africa's longest, instead claiming the kob migration is longer. Their 'straight line distance', however, is actually an estimate of how much ground their one collared kob covered $(825 \mathrm{~km})$. The appropriate comparison metric for the round-trip zebra migration is therefore the sum of the northbound and southbound Distance columns in Table 1 of Naidoo et al., which has a mean across individuals of $955 \mathrm{~km}$ (range 735-1170), with five of seven collared zebra covering greater distances than the kob individual.

Schapira et al. describe a migration that, as with Serengeti wildebeest, involves animals moving in an approximately circular manner, with similar start and end points. In contrast, the zebra migration has two spatially separate endpoints where animals spend prolonged periods: a dry season range along the Chobe River in Namibia and Botswana, and a wet season range in or near Nxai Pan National Park in Botswana (Fig. 1). It is this straight-line distance between geographical endpoints that is approximately $250 \mathrm{~km}$, hence our estimate that zebra cover $500 \mathrm{~km}$ in a straight line. The equivalent straight-line distance between endpoints in Schapira et al. is
RoBIN NAIDOO (Corresponding author) World Wildlife Fund, Conservation Science Program 1250 24th Street NW, Washington DC 20037, USA. E-mail robin.naidoo@wwfus.org

Michael CHASE and KeLLy LANDEN Elephants without Borders, Kasane, Botswana

PIeT BeYTell and PIeRRe DU PREeZ Directorate of Natural Resource Management, Ministry of Environment and Tourism, Windhoek, Namibia

Greg Stuart-Hill and Russell Taylor WWF in Namibia, Natural Resources Planner, Windhoek, Namibia

\section{References}

Harris, G., Thirgood, S., Hopcraft, G., Cromsigt, J.P.G.M. \& BERGER, J. (2009) Global decline in aggregated migrations of large terrestrial mammals. Endangered Species Research, 7, 55-76.

Naidoo, R., Chase, M.J., Beytell, P., Du Preez, P., Landen, K., StUART-Hill, G. \& TAYLOR, R. (2016) A newly discovered wildlife migration in Namibia and Botswana is the longest in Africa. Oryx, $50,138-146$.

Schapira, P., Monica, M., Rolkier, G.G. \& Bauer, H. (2016) Wildlife migration in Ethiopia and South Sudan longer than 'the longest in Africa': a response to Naidoo et al. Oryx, http://dx.doi.org/ $10.1017 /$ So030605316000363. 